\title{
Asteroseismology and evolution of EHB stars
}

\author{
R. H. Østensen \\ Instituut voor Sterrenkunde, K.U.Leuven, Celestijnenlaan 200D, B-3001 Leuven, Belgium
}

\begin{abstract}
The properties of the Extreme Horizontal Branch stars are quite well understood, but much uncertainty surrounds the many paths that bring a star to this peculiar configuration. Asteroseismology of pulsating EHB stars has been performed on a number of objects, bringing us to the stage where comparisons of the inferred properties with evolutionary models becomes feasible. In this review I will outline our current understanding of the formation and evolution of these stars, with emphasis on recent progress. The aim is to show how the physical parameters derived by asteroseismology can enable the discrimination between different evolutionary models.
\end{abstract}

Individual Objects: V361 Hya, V1093 Her, DW Lyn, V391 Peg, Balloon 090100001, KL UMa, NY Vir, V338 Ser, LSIV-14ำ 116 , HD 188112

\section{Introduction}

Let me first clarify the basic terminology with respect to EHB stars, which can sometimes be confusing as the terms EHB and sdB are often used to label the same stars. The terms Extended Horizontal Branch or Extreme Horizontal Branch have been used interchangeably to describe the sequence of stars observed to lie bluewards of the normal Horizontal Branch stars in globular clusters, and also in temperature/gravity plots of hot field stars. The EHB feature was first described and associated with field $\mathrm{sdB}$ and $\mathrm{sdO}$ stars by Greenstein \& Sargent (1974). Now, EHB stars are taken to mean core helium burning stars with an envelope too thin to sustain hydrogen burning. It is also understood that not all sdB stars are EHB stars. In particular, if a star loses its envelope without the core reaching the mass required for the helium flash, its cooling track can take it through the sdB domain on its way to become a helium core WD. The $\mathrm{sdB} / \mathrm{sdO}$ terms are used to describe the spectroscopic appearance and do not presume any particular evolutionary stage. Several subclassification schemes have been used, but most common nowadays is the one introduced by Moehler et al. (1990). This scheme names as sdB stars those of the hot subdwarfs showing $\mathrm{Hel}$ absorption lines, as sdO stars those showing Hell, and as sdOB stars those showing features of both. Additionally the terms $\mathrm{He}-\mathrm{sdB}$ and $\mathrm{He}-\mathrm{sdO}$ are used to describe stars in which the helium lines dominate over the Balmer lines. The EHB forms a sequence of stars from the coolest sdBs to the sdOB domain, and it is clear that most stars given this classification are in fact EHB stars. For the He-rich objects a coherent picture has yet to emerge.

The current canonical picture of the EHB stars was mostly established by Heber (1986), in which the EHB stars are helium core burning stars with masses close to the core helium flash mass of $\sim 0.47 \mathrm{M}_{\odot}$, and an extremely thin hydrogen envelope, too thin to sustain hydrogen burning (no more than $1 \%$ by mass). It is understood that they are post red giant branch 
(RGB) stars that have started core helium burning in a helium flash before or after the envelope was removed by any of several possible mechanisms. The lifetime of EHB stars from the zero-age EHB (ZAEHB) to the terminal age EHB (TAEHB), when core helium runs out, takes between 100 and 150 Myrs. The post-EHB evolution will take them through the sdO domain directly to the white dwarf (WD) cooling curve without ever passing through a second giant stage. The time they spend shell helium burning before leaving the sdO domain can be up to 20 Myrs.

Although the future evolution of EHB stars after core He-exhaustion has always been presumed quite simple, the paths that lead to the EHB have always been somewhat mysterious. New hope that the evolutionary paths leading to the formation of EHB stars can be resolved has been kindled by the discovery that many of them pulsate, which has opened up the possibility of probing their interiors using asteroseismological methods. These pulsators are known as sdBV stars, and several distinct subclasses are now recognized (see the Asteroseismology section below). But in order to understand what questions asteroseismology can ask and answer, it is essential to understand the different paths that produce EHB stars. Only by understanding the evolutionary history of these stars is it possible to construct realistic models of their interiors which are needed for asteroseismology to be able to distinguish between the different formation scenarios. For this reason we will review the essential points of the Formation and Evolution first, after starting with a look at the observed properties of the hot subdwarf population in The Observed EHB below.

Besides the spectacularly rapid pulsations in the EHB stars, another factor that has contributed to the recent burst in interest in EHB stars is the realization that these stars are the main contributor to the UV-upturn phenomenon observed in elliptical galaxies. An excellent review of the UV upturn and the binary population synthesis models required to model this phenomenon can be found in Podsiadlowski et al. (2008). For a more in-depth review of the properties of all hot subdwarf stars, the exhaustive review by Heber (2009) is recommended.

\section{The Observed EHB}

Hot subdwarf stars were found in the galactic caps already by Humason \& Zwicky (1947). By the time Greenstein \& Sargent (1974) wrote their seminal paper, the number of such faint blue stars had grown to 189 , permitting a systemic study of the population. The PG survey (Green et al. 1986), which covered more than 10000 square degrees at high galactic latitudes, found that of 1874 UV-excess objects detected more than 1000 were hot subdwarfs, so these stars dominate the population of faint blue stars down to the PG survey limit $(B=16.5)$. Together with the large sample of subdwarfs detected in the HS survey and analyzed by Edelmann et al. (2003), these have provided a rich source of hot subdwarfs for observers to follow up, and new discoveries are still being made. The recent Sloan Digital Sky Survey (SDSS, Stoughton et al. 2002) also contains spectra of more than 1000 hot subdwarfs, but as the SDSS reaches much deeper than the PG survey, WD stars start to dominate around about $B=18$ as the thickness of the galactic disk is reached. The Subdwarf Database (Østensen 2006) catalogs about 2500 hot subdwarfs, with extensive references to the available literature.

Several surveys have attempted to tackle the question of the binary frequency on the EHB, but the matter is complicated by the many different types of systems EHB stars are found in. EHB stars with FGK companions are easily detected from their double-lined spectra or from IR excess. But EHB stars with WD or M-dwarf companions show no such features. When the orbital periods are sufficiently short, these systems can easily be revealed from their RV variations. Using the RV method, Maxted et al. (2001) targeted 36 EHB stars and found 21 binaries, all with periods less than 30 days. This gave a fraction of short period binaries of $60 \pm 8 \%$. Other surveys have found smaller fractions, but they have not constrained the sample to focus strictly on the EHB. From high-resolution VLT spectra of $76 \mathrm{sdB}$ stars from the SPY survey Lisker et al. (2005) found that 24 showed the signature of an FGK companion, 




Figure 1: The EHB in the $T_{\text {eff }}-\log g$ plane as observed by the Bok-Green survey (Green et al. 2008). The symbols mark observed stars with the size indicating the helium abundance. The theoretical zero age HeMS is shown for a wide range of masses. Models from Paczyński (1971) with masses of 0.5, 0.7, 0.85, $1,1.5$ and $2 \mathrm{M}_{\odot}$ are marked with $*$ symbols (starting from low $T_{\text {eff }}$ ). More recent models from Kawaler \& Hostler (2005) are shown for $M_{*}=0.41,0.43, \ldots 0.57 \mathrm{M}_{\odot}$ marked with + symbols, and the zero age and terminal age EHB for the $0.47 \mathrm{M} \odot$ core models are also drawn. For the latter, four evolutionary tracks with different envelope thicknesses $\log M_{e} / M_{*}=-3.5,-3,-2.5,-2$ are drawn (starting from high $\log g$ ).

none of which show any RV variability. Napiwotzki et al. (2004) reported that of $46 \mathrm{sdB}$ stars in the same sample, 18 (39\%) were RV variable. Clearly, the binary fraction in EHB stars is much higher than for normal stars, but an accurate number has yet to be established.

Most recently, Green et al. (2008) have presented a uniform high signal-to-noise lowresolution survey of a large sample including most known hot subdwarf stars brighter than $V=14.2$, using the university of Arizona $2.3 \mathrm{~m}$ Bok telescope (hereafter referred to as the Bok-Green or BG survey). From this large sample the clearest picture of the EHB to date emerges (Figs. 1 and 2). Most stars in the diagram are clearly well bound by EHB models for a narrow mass distribution. Most of the remaining stars are consistent with post-EHB models, but could also fit core helium burning objects with higher than canonical masses. The most helium rich objects, however, appear to form their own sequence, which cannot be explained by canonical EHB models. The tail of the regular horizontal branch reaches into the diagram from the upper right, but is separated from the EHB by a substantial gap, as first noted by Newell (1973). Although the details of this survey are still under analysis, several new features have been noted. The sequence of He-rich objects around $40 \mathrm{kK}$ is not compatible with current evolutionary scenarios, since post-EHB and post-RGB objects pass too rapidly through this region of the $T_{\text {eff }}-\log g$ plane to produce the observed clustering, but the late hot flasher scenario (discussed in the next section) holds some promise.

The large group of stars below the helium main sequence (HeMS) is more problematic as no type of star should be able to stay in this position in the $T_{\text {eff }}-\log g$ plane more than briefly, and no clustering should occur. The feature was also noted by Stroeer et al. (2007), but it cannot be ruled out that this is an artifact of the models, as many of these stars appear to have significant amounts of CNO processed material in their atmospheres, and the NLTE models used do not account for this. Another remarkable feature appears when looking at the distribution of short period binaries in the $T_{\text {eff }}-\log g$ plane (Fig. 2). In particular, the incidence of such binaries appears to be much smaller at the hot, high gravity end of the 


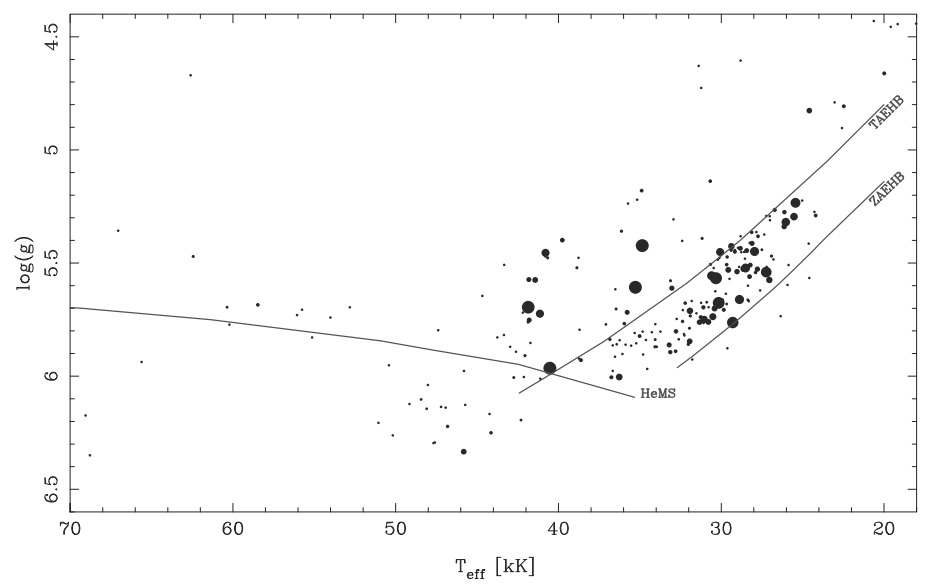

Figure 2: The same as Fig. 1, but with the symbol size indicating the dispersion in radial velocity. The EHB stars with the highest velocity variations appear to be concentrated at lower gravities on the EHB. sdB+FGK stars are not included here, due to difficulties in reliably disentangling such composite spectra.

EHB strip than among the cooler subdwarfs. This can be understood in terms of the relative efficiency of the common envelope ejection channel (CE, see next section) that produces short period binaries versus the other channels producing long period systems or single stars. It would appear that the CE channel is significantly less effective in removing the envelope than the other channels.

\section{Formation and evolution}

As binary interactions are a key for understanding the formation of EHB stars, I will attempt a short introduction here. Mass transfer during close binary evolution is well understood, although there are still some unknown factors. While the full picture is exceedingly complex, and would take far too much time to describe here, I will try to outline some of the most important possibilities.

\section{Losing the Envelope}

There are two fundamental evolutionary paths, and which one a system enters depends only on the mass ratio of the system. If the expanding mass donor is more massive than the accretor, the orbit will shrink catastrophically and the system enters a common envelope (CE) phase. As the orbit shrinks further due to friction, orbital energy is deposited in the envelope, spinning it up. When sufficient energy is deposited the envelope is ejected. Stars with an initial main sequence (MS) mass below about $1.8 \mathrm{M}_{\odot}$ can ignite helium in a core flash before the tip of the RGB, and if the envelope is ejected at the right time the result is an EHB star with a mass close to the flash mass of $\sim 0.47 \mathrm{M}_{\odot}$, and a very close low-mass companion. If the envelope is ejected before the core reaches the required mass, the core never ignites helium and the star will not settle on the EHB, but continues to contract and ends up as a helium core white dwarf. The close sdB+WD binary HD 188112 (Heber et al. 2003) is a particular point case, as the Hipparcos parallax together with the observed spectroscopic surface gravity clearly constrain the mass of the subdwarf component to be below the core 
helium burning limit. If the MS mass is higher than about $2.0 \mathrm{M}_{\odot}$ the star will ignite helium non-degeneratively well before the core reaches the mass required for the helium flash. If the envelope is subsequently ejected on the tip of the RGB the outcome would be an EHB star which could have a mass as low as $0.33 \mathrm{M}_{\odot}$.

On the other hand, if the companion is more massive than the red giant donor filling its Roche lobe, the orbit expands and no CE is formed. In this stable Roche lobe overflow (RLOF) scenario the orbital period can end up as long as 2000 days. As with CE ejection, if the red giant starts out with a mass below the mass required for the helium flash to occur, the mass transfer must happen close to the tip of the RGB, and the core of the giant becomes an EHB star with a mass close to $0.47 \mathrm{M}_{\odot}$. If it is too massive, non-degenerate helium burning starts earlier, and the result is an sdB star with a mass between 0.33 and $1.1 \mathrm{M}_{\odot}$ ( Han et al. 2000).

\section{The Problematic Singles}

A significant number of sdB stars are definitely single stars, and their formation is the most problematic and controversial. While post-CE systems leave behind a close binary that is easily detectable from the radial velocity (RV) variations, post-RLOF binaries have such long periods that it requires very long term efforts with high precision spectroscopy to detect them. Up to now there are no detections of any such orbits. However, long term asteroseismic monitoring can detect orbitally induced variations in the pulsation period with much higher precision than can be done from spectroscopy. The clearest case yet is V391 Peg where the modulation of the pulsations are consistent with a planet with a mass $(M \sin i)$ of $3.2 \mathrm{M}_{\text {Jupiter }}$ in an orbit with period of 1170 days (Silvotti et al. 2007). While the planet might have entered the outer layers of the envelope of the red giant before the envelope was lost, the current orbit is too wide for it to have been responsible for the actual envelope ejection.

Several scenarios have been proposed that may produce single EHB stars. It is well known that RGB stars lose significant mass in the form of a stellar wind as they expand and their surface gravity becomes extremely low. D'Cruz et al. (1996) computed evolutionary models of RGB stars with mass loss parameterized by the Reimers efficiency $\eta_{R}$. They found that the observed distributions of HB and EHB stars can be explained "so long as nature provides a broad enough distribution in $\eta_{R}$ ". However, the actual physics behind the large variation in the mass loss efficiency remains unexplained. Another possibility is the merger of two He-core WD stars, first proposed by Webbink (1984). Saio \& Jeffery (2000) have shown that models for such a merger can successfully predict the behaviour of the pulsating helium star V652 Her, demonstrating the feasibility of the merger scenario. Such extreme helium stars will evolve to become hot helium rich subdwarfs located close to the HeMS. However, a remaining problem with merger models is that they invariably leave behind rapidly rotating objects. So far, no single hot subdwarf has demonstrated more than moderate rotational velocities from high resolution spectroscopy.

Another possibility is that CE ejection can be triggered by a giant planet that evaporates in the process (Soker 1998). Nelemans \& Tauris (1998) demonstrated in the context of white dwarf evolution that there are clear domains in initial orbital period versus planetary mass, where the planet ejects the envelope and is disrupted as it fills its own Roche-lobe after the spiral in. The final rotation period of the remaining helium core is not affected by this process, as the planet transfers almost all of its angular momentum to the envelope before its ejection. A final possibility for the formation of single hot subdwarfs, also noted by Nelemans \& Tauris (1998) in the context of formation of undermassive white dwarfs, is a variation of the RLOF mechanism. If the envelope is transferred onto an accretor that is already a massive white dwarf, an asymmetric accretion induced collapse may produce a high velocity neutron star which escapes the system. If the companion is sufficiently massive to explode as a supernova, Marietta et al. (2000) have computed that the explosion itself 
impacts 1000 times more energy on the envelope than its binding energy, easily stripping the giant to the core. If the core is massive enough for helium burning, and the SN explosion sufficiently asymmetric, the remnant of the mass donor would end up as a single EHB star. In both cases the disruption of such a binary system would leave the EHB star with an unusual galactic orbit, which should be observable at least in a sufficiently large sample.

\section{To Flash or Not to Flash}

If an RGB star loses its envelope before the core has reached the mass required for the helium flash, the core will contract and heat up, before cooling as a helium core WD. The tracks calculated for such flashless post-RGB evolution covers a wide span in temperatures, with models around $0.2 \mathrm{M}_{\odot}$ passing through the cool end of the EHB, and the remnants with masses close to the helium flash mass reaching temperatures up to $100 \mathrm{kK}$ (Driebe et al. 1999).

A borderline case exists when the mass is just on the limit for the helium core flash to occur. Then the flash can happen after the RGB stage, while the core is either on its way to or on the actual WD cooling curve. Such models are known as hot flashers, and the eventual outcome depends on the exact stage at which the helium flash occurs. If ignition occurs before the turning point on the WD cooling track (early hot flashers), the remaining $\mathrm{H}$-burning shell produces a sufficient entropy barrier to prevent the convection zone produced by the helium flash to reach the surface (Iben 1976). But if helium ignites on the actual WD cooling curve, any remaining shell $\mathrm{H}$-burning is too weak to prevent the convection zone reaching the envelope. The envelope hydrogen is then mixed into the core and quickly burnt (Sweigart 1997), and CNO processed material is transported to the outer layers in a flash mixing process. Such late hot flashers are predicted to end up with an atmosphere almost totally devoid of hydrogen and with observable CNO lines in their spectra.

Recently, Miller Bertolami et al. (2008) have performed extensive simulations of late hot flashers in order to determine how well models can reproduce observations. They predict that the core flash cycles should take place in the region above the HeMS where the strip of helium rich subdwarfs are concentrated (Fig. 1). However, the core flash phase lasts less than 2 Myr, after which the stars settle close to the HeMS, for a regular EHB lifetime of at least $66 \mathrm{Myr}$. Observations do not support such a concentration of objects at this location in the $T_{\text {eff }}-\log g$ plane. To resolve this they propose that some remaining hydrogen could have survived the mixing and should slowly diffuse to the surface, effectively pulling the star up toward the cooler region of the EHB.

Another very recent development was presented by Politano et al. (2008). They have extended the classic common envelope ejection mechanism to include the case when a lowmass MS star or brown dwarf merges with the helium core, in order to produce single EHB stars. However, as with the helium white dwarf merger scenario, the problem remains that the products end up spinning close to break-up velocity. Since a rapidly rotating subpopulation of sdB stars has yet to be found, this channel is only of marginal interest, unless a way to eject the envelope without spinning up the core can be found.

\section{Asteroseismology}

The first evidence of rapid pulsations in EHB stars were reported by Kilkenny et al. (1997), after their detection of multiperiodic pulsations in V361 Hya. The V361 Hya stars span the hot end of the EHB strip from about 28 to $34 \mathrm{kK}$, and pulsate in p modes of low / orders and with photometric amplitudes up to $6 \%$. The pulsation periods range between 100 and $400 \mathrm{~s}$, and 40 such stars are known in the literature to date (Oreiro et al. 2009). One of these, V338 Ser, has periods reaching almost 10 minutes, but stands out as it sits well above the EHB (Fig. 3), being most likely in a post-EHB stage of evolution. 


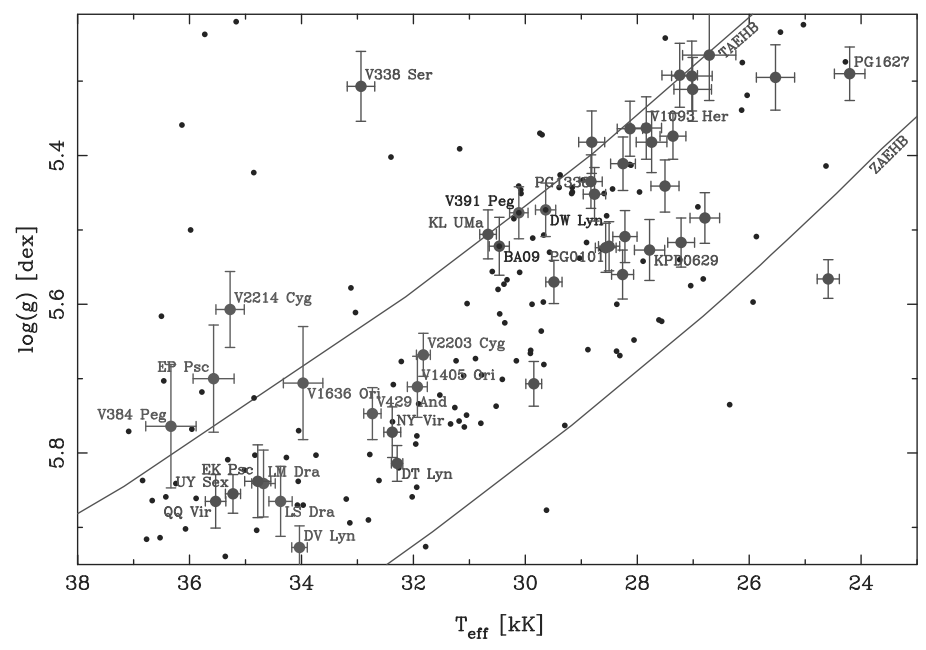

Figure 3: Section of the $T_{\text {eff }}-\log g$ plane where the EHB stars are located. Pulsators with temperatures and gravities in the BG survey are marked with big symbols and error bars. Small symbols without error bars are stars not observed to pulsate. In the online version the colours indicate short period pulsators with (green) and without (red) published asteroseismic solution, long period pulsators (magenta) and hybrid pulsators (blue core).

It took several years from the announcement of the first sdB pulsator until it was realized that the same group of stars are subject to longer period pulsations as well. Green et al. (2003) published the discovery of pulsations in $\mathrm{V} 1093 \mathrm{Her}$, with periods between one half and two hours, and reported that as many as $75 \%$ of sdB stars cooler than $30 \mathrm{kK}$ display some level of pulsations at these periods. The V1093 Her stars span the EHB from the coolest sdBs at around $24 \mathrm{kK}$ up to the domain of the V361 Hya stars (Fig. 3). The pulsations were identified with high radial order g modes by Green et al. (2003), and their amplitudes are very low, typically $0.2 \%$ or less. Although such low level pulsations are common in V1093 Her stars, and have been detected in at least 30, only a few have been studied in detail due to the long time-base and high precision required to detect and resolve their modes.

Even more recently, Schuh et al. (2006) realized that a known V361 Hya star, DW Lyn, was actually displaying the $\mathrm{g}$ modes of a V1093 Her star simultaneously with $\mathrm{p}$ modes of a V361 Hya star. As noted by the authors, the four V361 Hya stars DW Lyn, V391 Peg and Balloon 090100001 (BA09 in Fig. 3 and hereafter), and KL UMa, form a compact group closer to the domain of the V1093 Her stars than the remaining V361 Hya stars. Hybrid DW Lyn type pulsations have now been detected in both V391 Peg and BA09 as well, but appear to be absent in KL UMa. Intriguingly, KL UMa also stands out as the only binary of the quartet (O'Toole et al. 2004).

A fourth type of pulsations was noted in the He-sdB star LSIV $-14^{\circ} 116$ by Ahmad \& Jeffery (2005). They detected pulsations with amplitudes at the $1 \%$ level and periods around 15 minutes. The atmospheric parameters reported by the authors, $T_{\text {eff }}=32.5 \mathrm{kK}$, $\log g=5.4$ dex places the star just above the EHB strip in the $T_{\text {eff }}-\log g$ diagram, well surrounded by $\mathrm{V} 361 \mathrm{Hya}$ stars. With a supersolar helium abundance, $\log (\mathrm{He} / \mathrm{H})=-0.6$, this star represents a different evolutionary state than the regular EHB pulsators. Up to now this star remains unique, but since stars with the atmospheric properties of LS IV $-14^{\circ} 116$ are extremely rare it is too early to tell whether pulsations in stars with similar atmospheric parameters are 
common or rare.

A fifth and final class of pulsations in hot subdwarf stars was discovered by Woudt et al. (2006) in the hot sdO binary $\mathrm{J} 17006+0748$, but this will not be discussed here as this star is very far from the EHB region.

\section{Driving the Beat}

Pulsations in sdB stars were predicted to occur by Charpinet et al. (1996) at about the same time as the first pulsators were discovered by Kilkenny et al. (1997). The driving mechanism is due to an opacity bump caused by iron group elements (Charpinet et al. 1997). This mechanism is inefficient at solar metallicity, but gravitational settling and radiative levitation can work together to locally enhance metals in a driving zone in the envelope. This $\kappa$ mechanism has been successfully invoked to explain both the p-mode pulsations in V361 Hya stars and the g-mode pulsations in V1093 Her stars (Fontaine et al. 2003).

While the first models by Fontaine et al. (2003) could produce unstable modes in the coolest sdB stars, there appeared to be a gap between one island of instability on the cool end of the EHB and one at the hot end. The pulsators at the hot end of the g-mode instability region remained problematic, and particularly so the hybrid DW Lyn type pulsators. Some relief to this problem was recently provided by Jeffery \& Saio (2007), with the application of improved opacity values from the OP project as well as the explicit consideration of nickel in addition to iron. The new models are sufficient to bridge the gap between the hot and cool EHB pulsators, and could also predict an island of instability in the sdO domain, close to the observed location of J17006+0748. However, these most recent calculations do not yet give a perfect description of the observed picture. More unstable modes are still found in models at the hot end of the EHB than on the cool end, while observations indicate that pulsations are more common in cool sdB stars. In fact, the problem is now more to explain why most EHB stars on the hot end of the strip do not pulsate, than why they do. Jeffery \& Saio (2007) speculated that the iron group element enhancements, which build up due to a diffusion process over rather long time scales, may be disrupted by the the atmospheric motions as pulsations build up to some level. They note that since p modes mostly involve vertical motion, while $g$ modes are dominated by horizontal motion, it is possible that $\mathrm{p}$ modes are more effective at redistributing the iron group elements out of the driving zone.

\section{Levitation does the Trick}

Detailed models of the internal structure of the EHB stars are critical for improving our understanding of the asteroseismic properties. The simplest models with uniform metallicities are not able to drive pulsations in these stars at all. Only with the inclusion of an iron opacity bump was it possible to find unstable modes (Charpinet et al. 1996). However, the periods predicted by these so-called second generation models have usually not been matched with observed periods to better than about one percent, while the observed periods have a precision that are an order of magnitude better. Efforts to improve the atmospheric models to include more of the various effects that can have significant impact on the pulsation spectrum are ongoing.

Important progress was reported by Fontaine et al. (2006), who clearly demonstrated the importance of properly including time-dependent diffusion calculations in order to predict pulsation frequencies and mode stability. Starting from a uniform distribution and solar iron abundance, they demonstrated that it takes several hundred thousand years for radiative acceleration and gravitational settling to produce sufficient iron in the driving region to create unstable modes. After about $1 \mathrm{Myr}$ there are no more changes with respect to which modes are driven and not, but the pulsation frequencies may still shift as the iron opacity bump builds up further. After about $10 \mathrm{Myr}$ iron reaches equilibrium in these models, and no 



Figure 4: Published asteroseismic solutions for ten V361 Hya stars. The left hand panel shows total mass plotted versus envelope mass, and the right hand panel shows envelope mass versus surface gravity. Note that only the optimal solution is shown even if the papers discuss several possible ones.

further changes are seen. Since the time to onset of pulsations is just $1 / 1000$ of the typical EHB lifetime, this does not help resolve the issue as to why only a fraction of the EHB stars pulsate.

Second generation models based on a pure hydrogen atmosphere on top of a simple 'hard ball' core approximation, to which an explicit iron abundance profile is added, have been used to derive sensible asteroseismic quantities for a number of V361 Hya stars (e.g. Charpinet et al. 2007). The adopted 'forward' method basically consists of constructing a large grid of models in the four dimensional parameter space spanned by the fundamental model parameters, effective temperature $T_{\text {eff, }}$ surface gravity $\log g$, total mass $M$, and envelope mass fraction $q(\mathrm{H})$. A minimization procedure is then invoked to find the model that best matches the observed periods.

To date, ten asteroseismic solutions computed with this method have been published. They were summarized in Randall et al. (2007) for the first seven, and in Fig. 4 the new solutions by van Grootel et al. (2008), van Spaandonk et al. (2008) and Charpinet et al. (2008) (discussed below) have been included. A feature of the asteroseismic modelling is that $T_{\text {eff }}$ is rather poorly constrained, and a better value can usually be provided from spectroscopy. The surface gravity, total mass, and envelope mass fraction, however, have very small associated errors in the asteroseismic solutions, so we plot only these in Fig. 4. The distribution of masses is not as concentrated around $0.47 \mathrm{M}_{\odot}$ as most canonical evolutionary models have presumed, but all points are well within the permitted ranges for synthetic populations considered by Han et al. $(2002,2003)$. Except for two outliers, all the stars appear to form a trend with envelope mass, $M_{\mathrm{e}}$, increasing with total mass, $M$. Although this feature has not been accounted for by evolutionary calculations, it could occur as a natural consequence of a higher core mass requiring more energy to remove the envelope. More disturbing is the lack of any clear trend in envelope mass versus surface gravity, as is clearly demanded for canonical EHB models. The scatter in the high gravity objects is easily explained by their spread in mass, and KL UMa fits well with the expected $M_{e} / \log g$ trend. But the unusually low envelope masses for BA09 and V338 Ser are hard to explain, and may indicate that the adopted models are too simplified to represent the seismic properties for these cases. 


\section{Ballooney}

The exceptional amplitude of the dominant period in BA09 has hinted towards a radial nature, and this was finally confirmed by Baran et al. (2008) by combining evidence from multicolour photometry and the radial velocity amplitude measured by Telting \& Østensen (2006).

Van Grootel et al. (2008) have successfully applied the forward method to BA09, demonstrating some peculiarities in the model predictions. Their optimal solution for the main mode, when using no constraints, is $I=2$, which is not reconcilable with the spectroscopic data. However, by imposing mode constraints from multicolour photometry they do find asteroseismic solutions that agree with all observational data. Curiously, the physical parameters for the constrained and unconstrained fits are almost identical, even if the mode identification changes for half the modes considered. The authors conclude "Our primary result is that the asteroseismic solution stands very robust, whether or not external constraints on the values of the degree I are used." This peculiarity arises from the high mode density and the way the modes are distributed in period space. But the deeper cause of the problem is the low precision with which the second generation models predict pulsation periods. Models with a more detailed internal structure are therefore urgently needed in order to resolve this problem. It is a concern that the envelope mass fraction van Grootel et al. (2008) find $\left(\log M_{e} / M_{*} \simeq-4.9\right.$, regardless of which modes are which) is several hundred times lower than what any EHB model would predict for such a low-mass star at this position in the $T_{\text {eff }}-\log g$ diagram. With such a thin envelope all models put the star close to the HeMS for a core helium burning star. The authors' suggestion that the star is in a post-EHB stage of evolution is beyond canonical theory, as only models with substantial hydrogen envelopes evolve to lower gravities before moving off to the sdO domain as the core starts to contract (see Fig. 1).

The envelope mass discrepancy is even more severe in the asteroseismic results for V338 Ser (van Spaandonk et al. 2008), whose best fitting model (number 4 of 5 presented) has an envelope mass fraction $\log M_{e} / M_{*}=-5.78$ ! While the authors seem to prefer an even more extreme value of -6.22 for a model with a slightly poorer fit, in order to obtain a mass of $0.561 \mathrm{M}_{\odot}$ rather than the unusually high mass of $0.707 \mathrm{M}_{\odot}$, the high-mass solution might be the most interesting. For if the exceptionally high mass is real, evolutionary calculations would place V338 Ser in a core helium burning stage, not in a post-EHB stage as would be the case if its mass was around $0.5 \mathrm{M}_{\odot}$. But as for BA09, evolutionary models demand an envelope mass fraction more than 1000 times higher than found by van Spaandonk et al. (2008), in order to find V338 Ser at the observed $\log g$.

With a recent update of the forward modelling code, Charpinet et al. (2008) have produced a very convincing model for the eclipsing binary system NY Vir. This star has been particularly challenging since it is rapidly rotating, due to being in a tidally locked orbit with the close M-dwarf companion. The rotational splitting of modes with different $m$ produces a particularly rich pulsation spectrum. Charpinet et al. (2008) use asteroseismology to discriminate between three solutions from the binary orbit published by Vučković et al. (2007), and find that the intermediate model with a mass of $0.47 \mathrm{M}_{\odot}$ is clearly favoured. This solution is also the only one consistent with the log $g$ from the BG survey (Fig. 3).

Most recently, Telting et al. (2008) have presented the first study of line-profile variations in these stars based on high-resolution spectroscopy. Line profile variations of metal lines is a technique to directly determine the spherical harmonic order numbers $I, m$ of a pulsation mode, which is well established for various MS pulsators. To invoke this technique on the faint EHB stars requires substantial investments in terms of telescope time, which has prohibited its use up to now. With the preliminary results on the high amplitude pulsator BA09, Telting et al. (2008) clearly demonstrate that the I of the main mode must be either 0 or 1 . Again, the observational accuracy has advanced ahead of the theoretical models, as the standard modelling of such line profile variations are insufficient to properly account for the complex effects on the line profiles in the high temperature and gravity domain of the EHB stars. 


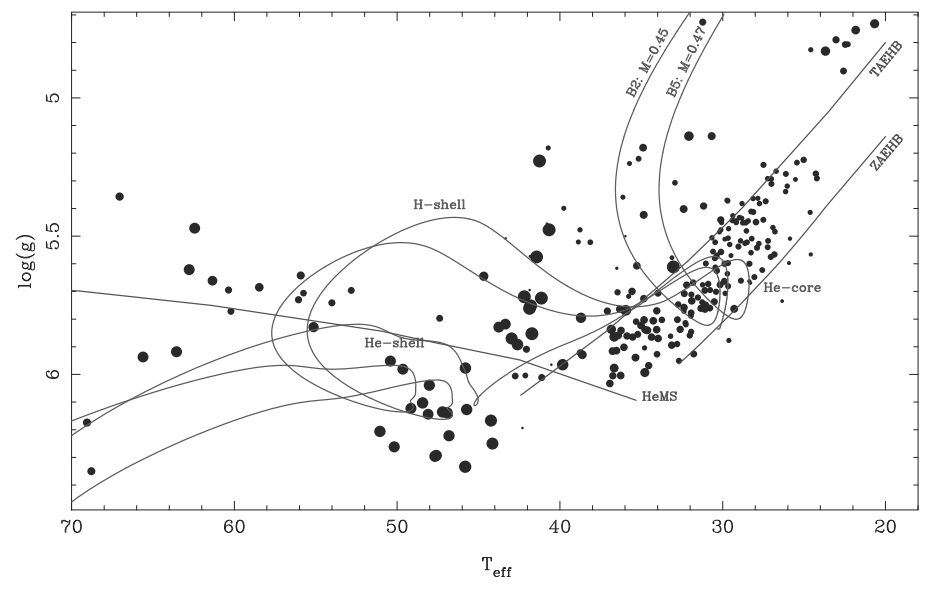

Figure 5: Same as Fig. 1, but with the evolutionary tracks for flashless post-RGB evolution from Hu et al. (2008) overplotted. The tracks evolve rapidly from the top of the plot to the ZAEHB as the envelope settles down on the helium burning core, and the stars spends most of their time (200 Myr) in the upward part of the loop at constant $T_{\text {eff }}$. After core helium exhaustion the star rapidly heats up, but then turns and moves back again for a several Myr long shell helium burning phase below the HeMS.

A final advancement that demonstrates with particular clarity the direction in which asteroseismology of EHB stars needs to move in order to progress, is the work of Hu et al. (2008). The authors have taken real evolutionary models that have been evolved from the ZAMS, through flashless helium ignition on the RGB, to the EHB by peeling of the envelope, and proceeded to compute the pulsation properties of these stars for a number of different configurations. By comparing these models with the classical post-flash models with similar surface parameters, they clearly show that the differences in internal structure from the two evolutionary paths produce significant differences in both the predicted pulsation periods, and with respect to which modes are excited or damped! Two of the flashless models of Hu et al. (2008) are plotted in Fig. 5 together with a classical post-flash EHB model. From an evolutionary population synthesis point of view it is interesting to note that the post-EHB tracks are substantially different. After the core has exhausted its helium and the star moves off the TAEHB the star briefly burns the remaining envelope hydrogen, before contracting and cooling down during a helium shell burning phase, which lasts much longer than in canonical models (up to $20 \mathrm{Myrs}$ ). This is long enough to produce a slight clustering of objects below the $\mathrm{HeMS}$ at temperatures between 45 and $50 \mathrm{kK}$, just where a substantial cluster of $\mathrm{He}-\mathrm{sdO}$ stars are observed.

\section{Conclusions}

Asteroseismology of EHB stars is a field in rapid progress, with exceptional challenges due to their complex formation paths. Much progress has been made on evolutionary models, but much remains to be done, particularly with respect to the formation of single EHB stars. Better models are also needed to reproduce the effects of the helium flash stage on the envelope composition, in order to reproduce the internal structure of EHB stars at the accuracy achieved by observational asteroseismology. Only when these advances are in place can asteroseismology reliably test the different formation scenarios. 
The low amplitudes and long periods make it very difficult to establish detailed pulsation spectra for V1093 Her stars, and even when it can be done the high mode density makes it difficult to assign modes to the observed frequencies. But $g$ modes are particularly interesting because they probe deep into the stellar interior. This is a significant challenge for the future due to the long time-base required to reliably determine the longer pulsation periods in these stars. The upcoming Kepler satellite mission (Christensen-Dalsgaard et al. 2007) provides an excellent opportunity if pulsators can be found within its field of view.

Acknowledgments. Special thanks to E.M. Green for kindly providing the detailed results from her 2008 article, which made it possible to reproduce the figures from that article together with evolutionary tracks. Without these data, Figs. 1, 2, 3, and 5 would have been a lot less informative. The author is supported by the Research Council of the University of Leuven under grant GOA/2008/04 and by the EU FP6 Coordination Action HELAS.

\section{References}

Ahmad, A., \& Jeffery, C. S. 2005, A\&A, 437, L51

Baran, A., Pigulski, A., \& O'Toole, S. J. 2008, MNRAS, 385, 255

Charpinet, S., Fontaine, G., Brassard, P., et al. 1997, ApJ, 483, L123

Charpinet, S., Fontaine, G., Brassard, P., \& Dorman, B. 1996, ApJ, 471, L103

Charpinet, S., Fontaine, G., Brassard, P., et al. 2007, CoAst, 150, 241

Charpinet, S., van Grootel, V., Reese, D., et al. 2008, A\&A, 489, 377

Christensen-Dalsgaard, J., Arentoft, T., Brown, T. M., et al. 2007, CoAst, 150, 350

D'Cruz, N. L., Dorman, B., Rood, R. T., \& O'Connell, R. W. 1996, ApJ, 466, 359

Driebe, T., Blöcker, T., Schönberner, D., \& Herwig, F. 1999, A\&A, 350, 89

Edelmann, H., Heber, U., Hagen, H.-J., Lemke, M., et al. 2003 A\&A, 400, 939

Fontaine, G., Brassard, P., Charpinet, S., et al. 2003, ApJ, 597, 518

Fontaine, G., Brassard, P., Charpinet, S., \& Chayer, P. 2006, Mem. S.A.It., 77, 49

Green, E. M., Fontaine, G., Reed, M. D., et al. 2003, ApJ, 583, L31

Green, E. M., Fontaine, G., Hyde, E. A., et al. 2008, ASP Conf. Ser., 392, 75

Green, R. F., Schmidt, M., \& Liebert, J. 1986, ApJSS, 61, 305

Greenstein, J. L., \& Sargent, A. I. 1974, ApJSS, 28, 157

van Grootel, V., Charpinet, S., Fontaine, G., et al. 2008, A\&A, 488, 685

Han, Z., Tout, C. A., Eggleton, P. P. 2000, MNRAS, 319, 215

Han, Z., Podsiadlowski, Ph., Maxted, P. F. L, et al. 2002, MNRAS, 336, 449

Han, Z., Podsiadlowski, Ph., Maxted, P. F. L, \& Marsh, T. R. 2003, MNRAS, 341, 669

Heber, U. 1986, A\&A, 155, 33

Heber, U., Edelmann, H., Lisker, T., Napiwotzki, R. 2003, A\&A, 411, L477

Heber, U. 2009, Annual Review of Astronomy and Astrophysics, 47, submitted

Hu, H., Dupret, M.-A., Aerts, C., et al., 2008, A\&A 490, 243

Humason, M. L., \& Zwicky, F. 1947, ApJ, 117, 313

Iben Jr, I. 1976, ApJ, 208, 165

Jeffery, C. S., \& Saio, H. 2007, MNRAS, 378, 379

Kawaler, S. D., \& Hostler, S. R. 2005, ApJ, 621, 432

Kilkenny, D., Koen, C., O'Donoghue, D., \& Stobie, R.S. 1997, MNRAS, 285, 640

Lisker, T., Heber, U., Napiwotzki, R., et al. 2005, A\&A, 430, 223

Marietta, E., Burrows, A., Fryxell, B., 2000, ApJS 128, 615

Maxted, P. F. L, Heber, U., Marsh, T. R., \& North, R. C. 2001, MNRAS, 326, 1391 
Miller Bertolami, M. M., Althaus, L. G., Unglaub, K., \& Weiss, A. 2008, A\&A, 491, 253

Moehler, S., Richtler, T., de Boer, K. S., et al. 1990, A\&ASS, 86, 53

Napiwotzki, R., Karl, C. A., Lisker, T., et al. 2004, Ap\&SS, 291, 321

Nelemans, G., \& Tauris, T. M. 1998, A\&A, 335, 85

Newell, E. B. 1973, ApJSS, 26, 37

Oreiro, R., Østensen, R. H., Green, E. M., \& Geier, S. 2009, A\&A, in press

$\varnothing$ stensen, R. H. 2006, Baltic Astronomy, 15, 85

O'Toole, S. J., Heber, U., \& Benjamin, R. A. 2004, A\&A, 422, 1053

Paczyński, B. 1971, Acta Astronomica, 21, 1

Podsiadlowski, Ph., Han, Z., Lynas-Gray, A. E., \& Brown, D. 2008, ASP Conf. Ser., 392, 15

Politano, M., Taam, R. E., van der Sluys, M., \& Willems, B. 2008, ApJ, 687, L99

Saio, H., \& Jeffery, C. S. 2000, MNRAS, 313, 671

Schuh, S., Huber, J., Dreizler, S., Heber, U., et al. 2006, A\&A, 445, L31

Silvotti, R., Schuh, S., Janulis, R., Solheim, J.-E., et al. 2007, Nature, 449, 189

Soker, N. 1998, AJ, 116, 1308

van Spaandonk, L., Fontaine, G., Brassard, P., \& Aerts, C. 2008, ASP Conf. Ser., 392, 387

Stoughton, C., Lupton, R.H., Bernardi, M., et al. 2002, AJ, 123, 485

Stroeer, A., Heber, U., Lisker, T., et al. 2007, A\&A, 462, 269

Sweigart, A. V. 1997, in "The Third Conference on Faint Blue Stars", eds. A. G. D. Philip, J. Liebert, R. Saffer, and D. S. Hayes, L. Davis Press, p. 3, arXiv:astro-ph/9708164

Telting, J. H., \& Østensen, R. H. 2006, A\&A, 450, 1149

Telting, J. H., Geier, S., Østensen, R. H., Heber, U., et al. 2008, A\&A, 492, 815

Vučković, M., Aerts, C., Østensen, R. H., et al. 2007, A\&A, 471, 605

Webbink, R. F. 1984, ApJ, 277, 355

Woudt, P. A., Kilkenny, D., Zietsman, E., et al. 2006, MNRAS, 371, 1497

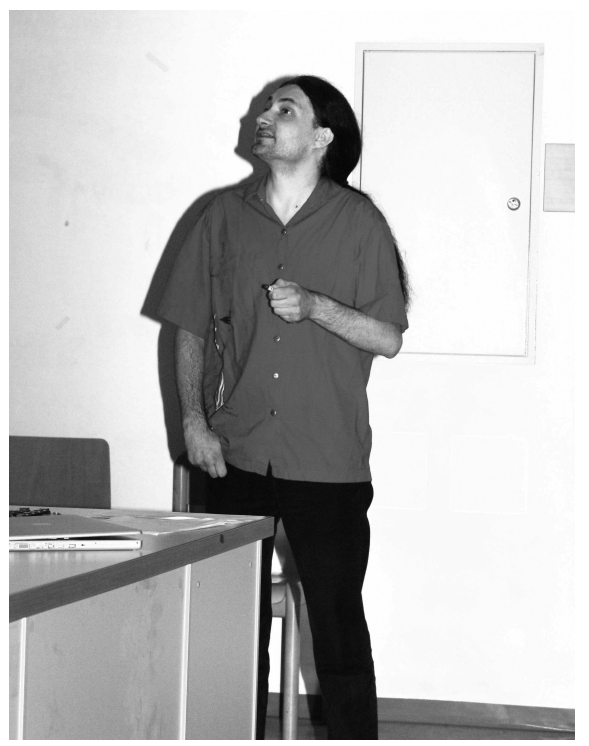

